\title{
Quenched charmonium spectrum on anisotropic lattices *
}

CP-PACS Collaboration : A. Ali Khan, S. Aoki, R. Burkhalter, ${ }^{\mathrm{a}, \mathrm{b}}$ S. Ejiri, ${ }^{\mathrm{a}}$ M. Fukugitac

S. Hashimoto, N. Ishizuka, ${ }^{a},{ }^{d}$ Y. Iwasaki, ${ }^{a}, b$ K. Kanaya, T. Kaneko, ${ }^{d}$ Y. Kuramashi, K.-I. Nagai,

M. Okamoto, M. Okawa, ${ }^{\mathrm{d}}$ H.P. Shanahan ${ }^{\mathrm{a} \dagger}$ Y. Taniguchi, A. Ukawa ${ }^{\mathrm{a}, \mathrm{b}}$ and T. Yoshiéa ${ }^{\mathrm{a}} \mathrm{b}$

${ }^{a}$ Center for Computational Physics, University of Tsukuba, Tsukuba, Ibaraki 305-8577, Japan

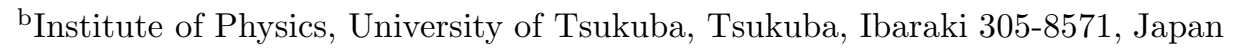

'Institute for Cosmic Ray Research, University of Tokyo, Kashiwa 277-8582, Japan

${ }^{\mathrm{d}}$ High Energy Accelerator Research Organization (KEK), Tsukuba, Ibaraki 305-0801, Japan

We present the results of quenched charmonium spectrum for S- and P-states, obtained by a relativistic heavy quark method on anisotropic lattices. Simulations are carried out using the standard plaquette gauge action and a meanfield-improved clover quark action at $a_{t}^{-1}=3-6 \mathrm{GeV}$ with the renormalized anisotropy fixed to $\xi \equiv a_{s} / a_{t}=3$. We study the scaling of our fine and hyperfine mass splittings, and compare with previous results.

\section{Introduction}

Conventional formalism of lattice QCD fails to describe heavy quarks because $a m_{Q} \gtrsim O(1)$ for charm and bottom quarks on current lattices with $a^{-1} \sim 1-3 \mathrm{GeV}$. In heavy hadrons, however, typical scale for the spatial momenta are comparable with that of light hadrons. A momentum with $O\left(m_{Q}\right)$ appears only in the time direction. Therefore, as proposed by Klassen, it is natural to adopt an anisotropic lattice with a small temporal lattice spacing with $a_{t} m_{Q} \ll 1$ for a lattice QCD description of heavy hadrons 1 [2. 2 .

In this report, we present results of our ongoing calculation on the quenched charmonium spectrum using the anisotropic method, which attempts to test the feasibility of this approach.

\section{Method and simulation parameters}

The gauge action we use is given by

$S_{g}=\beta \sum\left(1 / \xi_{0} P_{s s^{\prime}}+\xi_{0} P_{s t}\right)$,

where $\xi_{0}$ is the bare anisotropy. The renormalized anisotropy, defined by $\xi \equiv a_{s} / a_{t}$, can be de-

*Talk presented by M. Okamoto

†address after 15 Sept., 2000: Department of Biochemistry and Molecular Biology, University College London, London, England, UK
Table 1

Simulation parameters. Lattice spacing is fixed by the Sommer scale $r_{0}=0.5 \mathrm{fm}$.

\begin{tabular}{cccccccc}
\hline$\beta$ & $\xi$ & $\xi_{0}$ & $c_{s}$ & $c_{t}$ & $a_{s}^{r_{0}}[\mathrm{fm}]$ & $L^{3} \times T$ & $L a_{s}[\mathrm{fm}]$ \\
\hline 5.7 & 3 & 2.35 & 1.97 & 2.51 & 0.202 & $8^{3} \times 48$ & 1.61 \\
5.9 & 3 & 2.41 & 1.84 & 2.45 & 0.139 & $12^{3} \times 72$ & 1.67 \\
6.1 & 3 & 2.46 & 1.76 & 2.42 & 0.100 & $16^{3} \times 96$ & 1.60 \\
\hline
\end{tabular}

\begin{tabular}{cccccc}
\hline$\beta$ & $a_{t} m_{q 0}$ & $\zeta$ & iter/conf & \#conf & $c(0)$ \\
\hline 5.7 & 0.320 & 2.88 & 100 & 1000 & $1.009(6)$ \\
5.7 & 0.253 & 2.85 & 100 & 1000 & $1.009(7)$ \\
5.9 & 0.144 & 2.99 & 100 & 1000 & $0.991(8)$ \\
5.9 & 0.090 & 2.93 & 100 & 1000 & $0.991(8)$ \\
6.1 & 0.056 & 3.10 & 200 & 600 & $0.980(10)$ \\
6.1 & 0.024 & 3.03 & 200 & 600 & $0.982(10)$ \\
\hline
\end{tabular}

termined by Wilson loops. In this study, we use the result of [3] for $\xi\left(\xi_{0}, \beta\right)$.

For the quark action, we employ an $O(a)$ improved Wilson-type quark action on an anisotropic lattice [1, 2, 迎:

$$
\begin{aligned}
S_{f}= & \sum_{x}\left\{\bar{\psi}_{x} \psi_{x}\right. \\
& -K_{t}\left[\bar{\psi}_{x}\left(1-\gamma_{0}\right) U_{0, x} \psi_{x+\hat{0}}\right.
\end{aligned}
$$




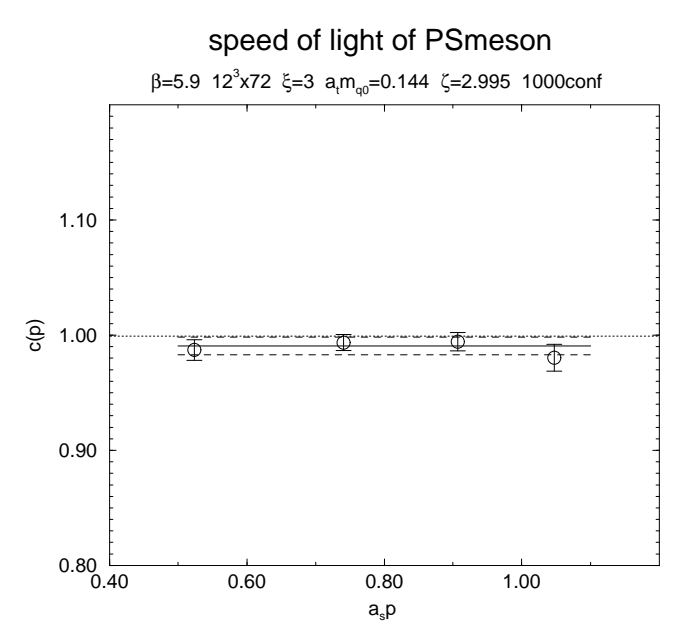

Figure 1. The speed of light and effective speed of light for $\eta_{c}$ at tuned $\zeta$ and $\beta=5.9$.

$$
\begin{aligned}
& \left.+\bar{\psi}_{x+\hat{0}}\left(1+\gamma_{0}\right) U_{0, x}^{\dagger} \psi_{x}\right] \\
& -K_{s} \sum_{i}\left[\bar{\psi}_{x}\left(1-\gamma_{i}\right) U_{i, x} \psi_{x+\hat{i}}\right. \\
& \left.\left.+\bar{\psi}_{x+\hat{i}}\left(1+\gamma_{i}\right) U_{i, x}^{\dagger} \psi_{x}\right]\right\} \\
& +i K_{s} c_{s} \sum_{x, i<j} \bar{\psi}_{x} \sigma_{i j} F_{i j}(x) \psi_{x} \\
& +i K_{s} c_{t} \sum_{x, i} \bar{\psi}_{x} \sigma_{0 i} F_{0 i}(x) \psi_{x}
\end{aligned}
$$

where $K_{s, t}$ and $c_{s, t}$ are the spatial and temporal hopping parameters and clover coefficients. The bare quark mass is given by

$a_{t} m_{q 0}=1 / 2 K_{t}-3 / \zeta-1$,

where $\zeta \equiv K_{t} / K_{s}$. We adopt the meanfieldimproved clover coefficients:

$c_{s}=\frac{1}{\left\langle U_{s}\right\rangle^{3}}, \quad c_{t}=\frac{1+\xi}{2} \frac{1}{\left\langle U_{s}\right\rangle\left\langle U_{t}\right\rangle^{2}}$,

where $\left\langle U_{s}\right\rangle=\left\langle P_{s s^{\prime}}\right\rangle^{1 / 4}$ with $P_{s s^{\prime}}$ the spatial plaquette and $\left\langle U_{t}\right\rangle=1$.

Our simulation parameters are summarized in Table 1. We study lattices with a fixed renormalized anisotropy $\xi=3$ and approximately the same spatial lattice size of about $1.6 \mathrm{fm}$. In order to study the charm quark, we simulate three lattices with the lattice cutoff in the range $a_{t}^{-1}=3-6$ $\mathrm{GeV}$.

The parameter $\zeta$ has to be tuned such that the relativistic errors are eliminated. For this purpose
Table 2

S- and P-state operators.

\begin{tabular}{ccccl}
\hline state & $J^{P C}$ & name & local & non-local \\
\hline${ }^{1} S_{0}$ & $0^{-+}$ & $\eta_{c}$ & $\psi$ & \\
${ }^{3} S_{1}$ & $1^{--}$ & $J / \psi$ & $\bar{\psi} \gamma_{i} \psi$ & \\
${ }^{1} P_{1}$ & $1^{+-}$ & $h_{c}$ & $\bar{\psi} \sigma_{i j} \psi$ & $\bar{\psi} \gamma_{5} \Delta_{i} \psi$ \\
${ }^{3} P_{0}$ & $0^{++}$ & $\chi_{c 0}$ & $\bar{\psi} \psi$ & $\bar{\psi} \sum_{i} \gamma_{i} \Delta_{i} \psi$ \\
${ }^{3} P_{1}$ & $1^{++}$ & $\chi_{c 1}$ & $\bar{\psi} \gamma_{i} \gamma_{5} \psi$ & $\bar{\psi}\left\{\gamma_{i} \Delta_{j}-\gamma_{j} \Delta_{i}\right\} \psi$ \\
${ }^{3} P_{2}$ & $2^{++}$ & $\chi_{c 2}$ & & $\bar{\psi}\left\{\gamma_{i} \Delta_{i}-\gamma_{j} \Delta_{j}\right\} \psi(\mathrm{E})$ \\
& & & & $\bar{\psi}\left\{\gamma_{i} \Delta_{j}+\gamma_{j} \Delta_{i}\right\} \psi(\mathrm{T})$ \\
\hline
\end{tabular}

preparatory simulations are performed at $\zeta=2.8$, 3.0 and 3.2. We measure the pseudoscalar meson mass at four lowest on- and off-axis momenta and perform a fit of form,

$E(p)^{2}=E(0)^{2}+c^{2}(p) p^{2}$

to extract the "speed of light", $c(p)$, using three or four lowest momenta. The tuning condition for $\zeta$ is $c(p=0)=1$ which requires the relativistic dispersion relation to be restored for small momenta. (For massless free fermions, $c(p=0)=1$ is satisfied at $\zeta=\xi$.) Results for $\zeta$ are summarized in Table $\mathbb{1}$.

Figure 1 shows a typical result for the effective speed of light $c_{\text {eff }}(p)=\sqrt{E(p)^{2}-E(0)^{2}} / p$, obtained by a simulation made with a tuned value of $\zeta$. The wide plateau indicates that the linearity of $E(p)^{2}$ in $p^{2}$ is well satisfied. The horizontal solid line is the result of $c(0)$ from a global fit according to (5), where the dashed lines indicate its error. We see that $c(0)$ is consistent with 1 . As summarized in Table 1, the condition $c(0)=1$ is confirmed to be satisfied within $1-2 \%$ with our values of $\zeta$.

\subsection{Measurements}

At each $\beta$, hadronic measurements are made on configurations separated by 100-200 iterations, at two values of $m_{q 0}$. See Table $\mathbb{1}$. These two quark masses are chosen such that the charm quark point can be interpolated.

We measure all S- and P-state mesons using both local and non-local operators compiled in Table 2. For the non-local operators we adopt an 


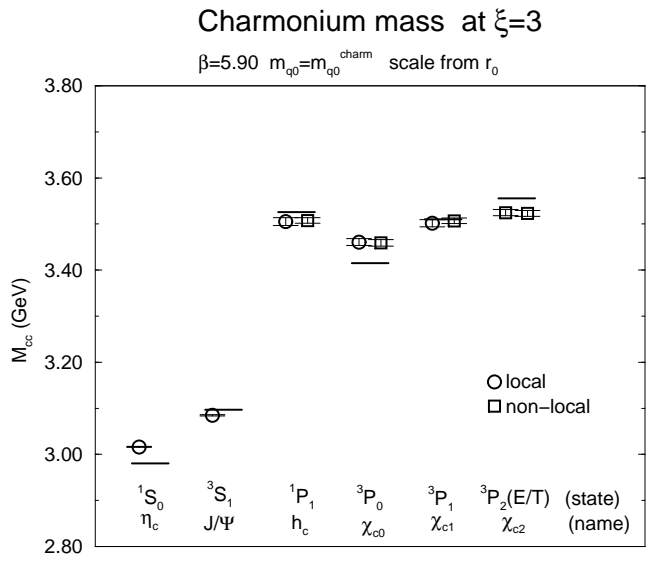

Figure 2. The charmonium mass spectrum at $\xi=$ 3 and $\beta=5.9$. The scale is fixed through the Sommer scale $r_{0}$.

Table 3

Preliminary continuum estimates of mass splittings and the experimental values.

\begin{tabular}{cccc}
\hline & $a_{s} \rightarrow 0$ & $a_{s} \rightarrow 0$ & Exp. \\
\hline scale & $r_{0}$ & ${ }^{1} P_{1}-S$ & \\
\hline$\Delta M_{J / \psi-\eta_{c}}[\mathrm{MeV}]$ & $65(1)$ & $82(2)$ & 117 \\
$\Delta M_{\chi_{c 1}-\chi_{c 0}}[\mathrm{MeV}]$ & $55(5)$ & $63(5)$ & 95 \\
$\Delta M_{\chi_{c 2}-\chi_{c 1}}[\mathrm{MeV}]$ & $19(7)$ & $21(7)$ & 46 \\
$\frac{\Delta M_{\chi_{c 2}-\chi_{c 1}}}{\Delta M_{\chi_{c 1}-\chi_{c 0}}}$ & $0.33(15)$ & $0.31(14)$ & 0.48 \\
\hline
\end{tabular}

exponentially smeared derivative sources:

$f_{i}(\mathbf{x})=A_{s} e^{-B_{s}|\mathbf{x}-\hat{i}|}-A_{s} e^{-B_{s}|\mathbf{x}+\hat{i}|} \quad(i=1,2,3),(6)$

where $A_{s}$ and $B_{s}$ are the smearing parameters. We extract meson masses by a single cosh fit. Errors are determined by a jack-knife method.

\section{Charmonium spectrum}

We fix the scale using either the Sommer scale $r_{0}=0.5 \mathrm{fm}$ or the $1^{1} P_{1}-1 \bar{S}$ splitting. The charm quark point is then defined by setting the spin averaged $1 \mathrm{~S}$ meson mass $(1 \bar{S})$. Figure 2 is a typical result for the charmonium mass spectrum obtained at $\beta=5.9$. In the following, we study the lattice spacing dependence of the spectrum. With the $O(a)$ improved action, the leading scaling violation is expected to be proportional to $a^{2}$.

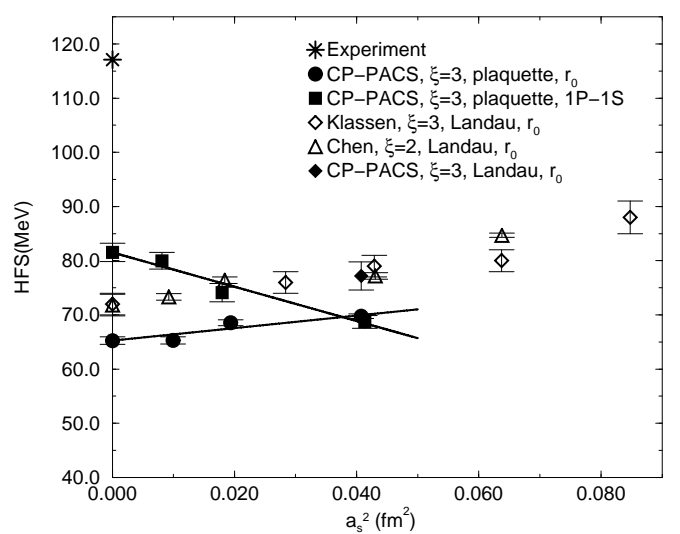

Figure 3. The scaling behavior of hyperfinesplitting $\Delta M_{J / \psi-\eta_{c}}$. Filled circle and square are our results with scale from the Sommer scale $r_{0}$ and the $1 P-1 \bar{S}$ splitting respectively. Open diamond and triangle are results of Ref. [2] and [5].

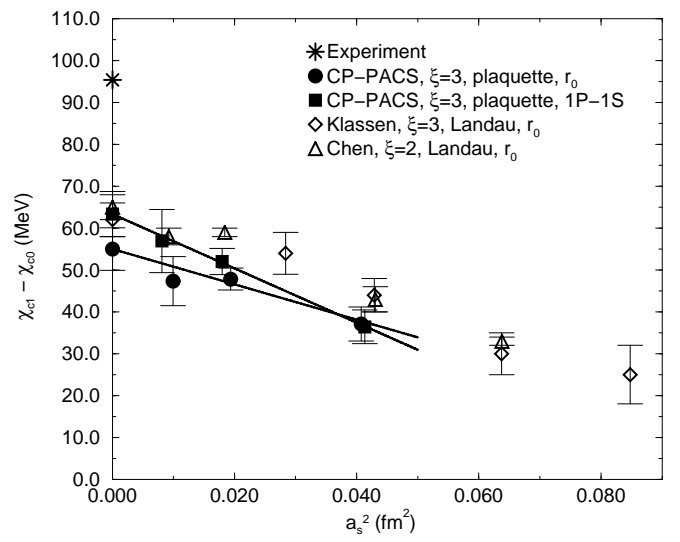

Figure 4. The scaling behavior of $\Delta M_{\chi_{c 1}-\chi_{c 0}}$.

\subsection{Hyperfine splitting}

Figure 3 is our result for the S-state hyperfinesplitting $J / \psi-\eta_{c}$ as a function of the lattice spacing. Results using the scales from $r_{0}$ and $1^{1} P_{1}-1 \bar{S}$ are presented. We find that, although our results for the hyperfine-splitting can be approximately fitted by a linear function of $a_{s}^{2}$, the two results from different scales lead to different values even in the continuum limit. This discrepancy should be regarded as a quenching artifact.

Plotted together are the previous lattice results by Klassen 20 and Chen 5] at $\xi=2$ and 3, using the same lattice action. A difference between our 


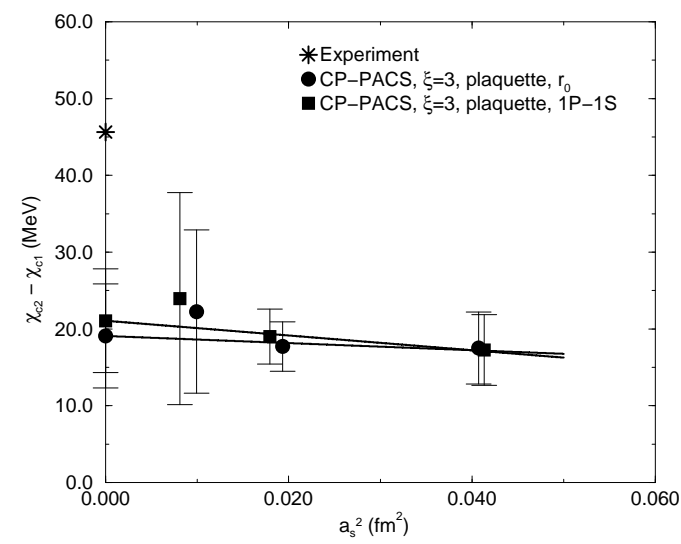

Figure 5. $\Delta M_{\chi_{c 2}-\chi_{c 1}}$.

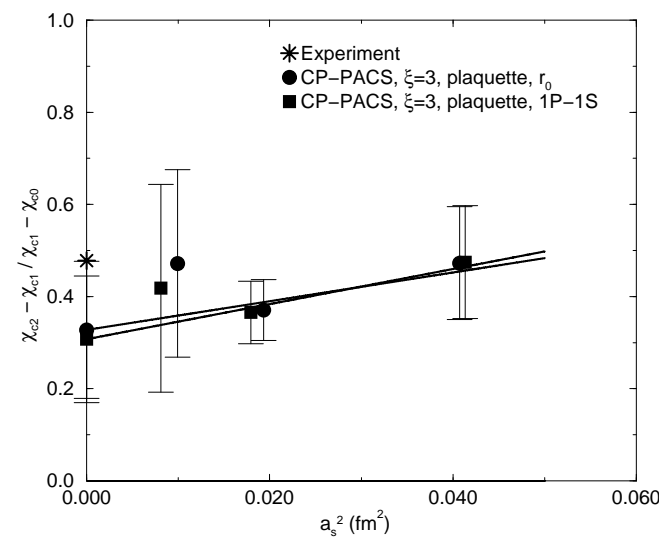

Figure 6. The ratio $\Delta M_{\chi_{c 2}-\chi_{c 1}} / \Delta M_{\chi_{c 1}-\chi_{c 0}}$.

simulation and those in [2] and [5] is the choice of the tadpole factor for the clover coefficients; we use the fourth root of the plaquette expectation value, while the mean link in the Landau gauge is used in [2] and [5]. To check the consistency, we also performed a simulation at $\beta=5.7$ using the Landau gauge mean link for the meanfield. As shown in Fig. 3 by a filled diamond, our result is consistent with those by Klassen.

We find that our result from the scale $r_{0}$ is approximately parallel to the other two results, and lead to a continuum limit which is about $10 \%$ lower than that of the others. The origin of this discrepancy is not clear to us at present.

\subsection{Fine structure}

Results for the P-state fine structure $\chi_{c 1}-\chi_{c 0}$ are shown in Fig. A. Although the scaling behavior of our results is similar to those of [2] and [5], the continuum limit deviates by about $2 \sigma$ for the data using the $r_{0}$ scale.

We also show the results for the $\chi_{c 2}-\chi_{c 1}$ splitting and the ratio $\chi_{c 2}-\chi_{c 1} / \chi_{c 1}-\chi_{c 0}$ in Figs. 5 and 6. No data from other groups are available for these quantities.

\section{Conclusions}

We have presented our results for the charmonium mass spectrum obtained on an anisotropic lattice with $\xi=3$. Our results for fine and hyperfine mass splittings are shown in Figs. 3 6. Our preliminary values from a continuum extrapolation linear in $a^{2}$ are summarized in Table 3 . We find that these results are much smaller than experimental values.

We also find that the results are quite sensitive to the choice of the clover coefficient. A naive continuum extrapolation, linear in $a^{2}$, does not resolve the discrepancy between different choices of the clover coefficient. We are now extending the simulation to a finer lattice, to examine the reliability of continuum extrapolations.

This work is supported in part by Grants-inAid of the Ministry of Education ( Nos. 10640246, 10640248, 10740107, 11640250, 11640294 , 11740162 12014202, 12304011, 12640253, 12740133). AAK is supported by JSPS Research for the Future Program (No. JSPS-RFTF 97P01102). SE, TK, KN, M. Okamoto and HPS are JSPS Research Fellows.

\section{REFERENCES}

1. T.R. Klassen, Nucl. Phys. B Proc. Suppl. 73 (1999) 918, hep-lat/9809174.

2. T.R. Klassen, unpublished.

3. T.R. Klassen, Nucl. Phys. B533 (1998) 557.

4. A.X. El-Khadra, A.S. Kronfeld and P.B. Mackenzie, Phys. Rev. D55 (1997) 3933.

5. P. Chen, hep-lat/0006019. 